\section{Technology of Fluoropolymers}

by Jiri George Drobny: CRC Press, Boca Raton, USA, 2nd edn, 2008, pp 225, Appendices and Index, ISBN: 978-1-4200-6317-2.

Price: EUR 114.99, USD 135.96

This is a relatively slim text but does cover all aspects of the technology of fluoropolymers. The book is intended to provide fundamental information in a systematic manner, to give easy access to specific properties and overviews of the topics within the individual chapters. This book does not claim to provide all of the details.

The introduction attempts to give the reader a feel for the background and the importance of fluoropolymers generally, while the second and third chapters cover the basic chemistry and properties of these polymers. The next four chapters really give more information of the processing and applications of fluoropolymers but separately, covering the main commercial materials, fluoroelas- tomers, aqueous dispersion fluoropolymers and the more obscure related materials. Fluoropolymers are all relatively inert materials but with generally less thermal stability and more reactivity as other elements or reactive sites are introduced. For this reason, there is a tendency for repetition from chapter to chapter - starting with descriptions of the more stable, less reactive fully fluorinated materials progressing through the same pattern of functionality that allows easier processing and reaction of polymers that are not fully fluorinated.

The chapters specifically on fluoroelastomers and on fluoropolymers in aqueous suspension, together with a chapter covering less commonly encountered materials (such as fluorinated acrylates and fluorinated polyurethanes) give substantial breadth to the coverage. Short chapters look at the effects of heat, radiation and environment on most of the fluoropolymer types, on the recycling of fluoropolymers and on safety and disposal but these are brief and with comparatively few references.
The final chapter of the book briefly covers 'New developments and current trends' with fluoropolymers. The new developments are covered by short sections on use of supercritical carbon dioxide for polymerisation, advanced polymer architecture technology, PFOAbased surfactants and novel elastomers. There are also brief sections on a range of processing developments. The developments in applications consider fluoropolymers in the automotive, aerospace and telecommunications industries but some of these considerations are extremely brief.

The main chapters include a lot of tabulated data and lots of references. There are also eight appendices with additional reference information and a reasonable index. While this book does not go into detail on the various topics, much basic property information is provided and there are the references to further sources of information.

\section{S. Holding}

